\title{
MicroRNA sequence and expression database
} Koray Dogan Kaya*1, Gokhan Karakulah² ${ }^{2}$ Cengiz Yakicier ${ }^{1}$ and Ozlen Konu ${ }^{1}$

Address: ${ }^{1}$ Department of Molecular Biology and Genetics, Bilkent University, Ankara, Turkey and ${ }^{2}$ Department of Medical Informatics, Institute of Health Sciences, Dokuz Eylul University, Izmir, Turkey

Email: Koray Dogan Kaya* - kkaya@bilkent.edu.tr

* Corresponding author

from BioSysBio 2007: Systems Biology, Bioinformatics and Synthetic Biology

Manchester, UK. II-13 January 2007

Published: 8 May 2007

BMC Systems Biology 2007, I(SuppI I):P29 doi:I0.1 186/1752-0509-I-SI-P29

This abstract is available from: http://www.biomedcentral.com/I752-0509/I?issue=SI

(c) 2007 Kaya et al; licensee BioMed Central Ltd.

\section{Background}

MicroRNAs, which are small ribonucleic acids that bind to 3'UTR regions of mRNAs by base complementation, play crucial roles in regulation of development and differention [1]. Herein we report on development of a web interfaced database, Bilkent University miRNA Sequence and Expression database [Figure 1; http://139.179.97.62/ nkoray] that integrates the species-specific mature miRNA sequence information and a set of associated public microarray data as tabular and graphical summaries suplemented with statistical analyses http://www.biocon ductor.org. The database also makes use of GO annotation data of miRNAs targets to determine the significance of GO term enrichment in a subset of miRNAs.

\section{Data and methods}

Bilkent University miRNA sequence and expression database was constructed using Mysql version 14.7 on Suse

\section{Billemtunirersity}

\section{mili:M sequence and expression database}

\footnotetext{
This database is constructed for observing the miRNA functions from the systems point of view. The database is comprising human, zebrafish, C . elegans and mouse mature miRNA data. Curres zombines mature miRNA dinucleotide properties to the public miRNA expression data and it links this relation to ontology data of their targets. The dinucleotide frequencies may reflect the genomic po aence function. After the selection of a dataset you may choose the set of miRNAs that bear the selected dinucleotide or the reverse set, or you may choose a completely different set by manual cli sheckboxes. Submission of this job will give the mean expression values as a graph for the conditions defined by the selected dataset. You may then retrieve ontology data related to the targets which statistically significant ontology terms. Or you can just deal with ontology of the targets just buy selecting an absent dinucleotide and an organism from sequence properties section
}

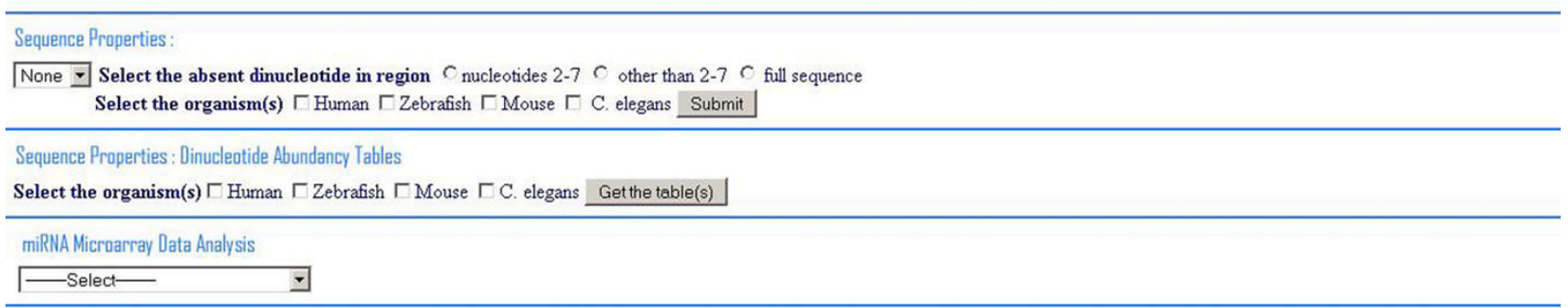

\section{Figure I}

The interface of miRNA sequence and expression database. 


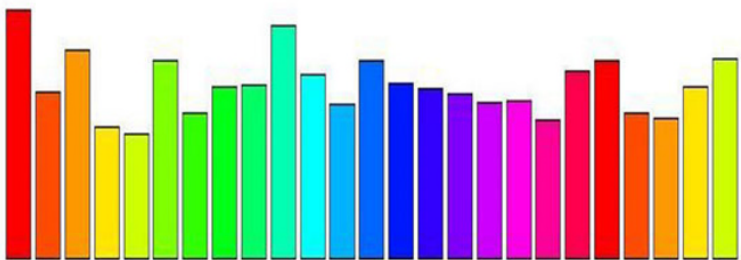

BM B H K Li LU Pa PI SM SQ Th FC Ly Co H.SS Ce a te A U Bt F SI Pl O

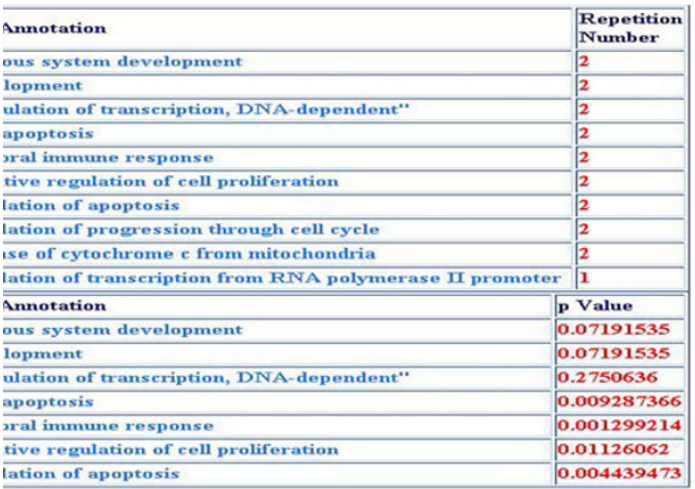

\section{Figure 2}

Left: Bar graph of microarray expression data for Hsa-mir-I5a and Hsa-mir- 16. Right: miRNA target GO annotation distribution and significantly enriched GO terms reported. One of the annotated targets were found to be BCL-2, a well known antiapoptotic protein [5].

Linux 10.0 server; the web interface implemented in HTML 4.0 combined with PHP version 4.4.0. Statistical calculations were performed using $\mathrm{R}$ package version 2.2.1. miRNA mature sequences from Homo sapiens, Mus musculus, Danio rerio and Caenorhabditis elegans were downloaded from miRBase of Sanger Institute database http://microrna.sanger.ac.uk/ version 8.2. Two independent microarray data sets $[3,4]$ were associated with human and mouse miRNA dinucleotide motif frequency, respectively. Human miRNA targets were extracted from Argonaute Database of Heildelberg http://www.ma.uniheidelberg.de/apps/zmf/argonaute/interface/. GO ontology data were linked with Argaonaute gene symbols and alias data from Entrez Gene Database http:// www.ncbi.nlm.nih.gov/entrez/query.fcgi? DB=gene.

\section{Results and conclusions}

The database allows for selection of miRNAs based on their dinucleotide properties and reports expression pattern of this particular set of sequences (Figure 2).

High expression of hsa-mir-15a and hsa-mir-16, known to be deleted in chronic lymphocytic leukemia, was detected in bone marrow and spleen (Figure 2a). Analysis of these miRNAs in terms of their targets resulted in significant representation of antiapoptosis and humoral immune response GO terms, both attributable to BCL-2 (Figure 2b). Although BCL2 is well known for its role in cell survival, there is no known direct relation of BCL-2 with immune response. Future studies involve integration of multiple species-specific gene expression data sets and implementation of correspondence analysis tools for multivariate analysis of sequence and expression data. The presented database will help understand miRNAs in a sys- tems biology context via integration of the sequence, expression, and functional attributes.

\section{References}

I. Bartel DP: MicroRNAs: genomics, biogenesis, mechanism and function. Cell 2004, I 16:28I-297.

2. Koray DK: Evaluation of microRNA sequence composition and expression. In M.Sc. thesis of Bilkent University Molecular Biology and Genetics Department, Ankara, Turkey; 2005.

3. Baskerville S, Bartel DP: Microarray profiling of microRNAs reveals frequent coexpression with neighboring miRNAs and host genes. RNA 2005, I I (3):24I-7.

4. Thomson JM, Parker J, Perou CM, Hammond SM: A custom microarray platform for analysis of microRNA gene expression. Nat Methods 2004, I(I):47-53.

5. Carney DA, Wierda WG: Genetics and molecular biology of chronic lymphocytic leukaemia. Curr Treat Options Oncol 2005, 6(3):215-25.
Publish with BioMed Central and every scientist can read your work free of charge

"BioMed Central will be the most significant development for disseminating the results of biomedical research in our lifetime. "

Sir Paul Nurse, Cancer Research UK

Your research papers will be:

- available free of charge to the entire biomedical community

- peer reviewed and published immediately upon acceptance

- cited in PubMed and archived on PubMed Central

- yours - you keep the copyright 